\title{
How Closely Should We Follow Gastric Cancer Patients Following Surgical Resection?
}

\author{
Sam S. Yoon, $\mathrm{MD}^{1,2}$ \\ ${ }^{1}$ Division of Surgical Oncology, Department of Surgery, Massachusetts General Hospital, Harvard Medical School, \\ Boston, MA; ${ }^{2}$ Department of Cancer Biology, University of Pennsylvania School of Medicine, Philadelphia, PA
}

The utility of intensive follow-up of patients with gastrointestinal malignancies following surgical resection has been a source of persistent debate. Most of the studies on this topic have been performed for patients with colorectal cancer, and the conclusions are mixed. In a meta-analysis of 8 randomized trials with 2923 colorectal cancer patients, more intensive follow-up was found to detect recurrences 5.9 months earlier and increased the proportion of recurrences amenable to surgical re-resection $(10.7 \%$ vs $5.7 \%){ }^{1}$ Curiously, overall mortality was improved for patients with more intensive surveillance (odds ratio 0.074, $P=0.01$ ), but cancer-related mortality was not different in the 2 groups.

There is significantly less randomized data on the follow-up of patients with gastric cancer. In this issue, Eom et al. from the National Cancer Center in Gyeonggi-do, Korea, report on the effectiveness of regular follow-up in their series of 1767 gastric cancer patients who underwent surgical resection. ${ }^{2}$ The follow-up regimen varied for early gastric cancers (defined at T1 tumors) and for late gastric cancers (defined at $\mathrm{T} 2$ or greater tumors), but included routine physical examinations, laboratories (CBC, LFTs, CEA, and CA-19-9), abdomen/pelvis computed tomography (CT) scans, chest X-rays, and yearly endoscopy. A total of 310 patients $(17.5 \%)$ developed recurrences, but median follow-up was not reported. Three-quarters of recurrences were detected by follow-up while one-quarter were detected following evaluation of symptoms. There was no survival difference between patients who had recurrences detected by follow-up studies versus those who had recurrences detected between follow-up visits, and the authors concluded that "the oncologic effectiveness of

(C) Society of Surgical Oncology 2010

Published Online: 2 December 2010

S. S. Yoon, MD

e-mail: syoon@partners.org regular follow-up after curative resection for gastric cancer was found to be unsatisfactory." Unfortunately, it is difficult to make conclusions on the effectiveness of follow-up when retrospectively analyzing patients who all received relatively intensive follow-up.

In order for intensive follow-up of patients to impact on subsequent survival, several conditions need to be met. First, a significant proportion of patients need to recur. Any benefit for intensive follow-up will be diminished if only a small percentage of patients actually recur. Second, the surveillance program must detect asymptomatic recurrences significantly earlier than without surveillance. If there is a short interval between detection of asymptomatic recurrence and the onset of symptoms, the potential benefit of initiating therapy early in recurrence is decreased. Third, there must be effective therapy for recurrent disease. There will be no effect of surveillance on survival if we have no effective therapy for recurrence. Finally, therapy for recurrent disease must be more efficacious for earlier recurrences compared with later recurrences.

In terms of the proportion of patients that recur, only $17.5 \%$ of patients in this study recurred, which is a relatively low percentage compared with Western series. The low percentage of patients who recurred is likely because the majority of patients had early-stage disease (although the stage of patients is not included in the article). In countries such as the United States where no endoscopic screening programs exist and patients generally present with later-stage disease, the percentage of patients who recur after potentially curative surgery is significantly higher, and thus the potential for identifying recurrences from intensive follow-up is greater. ${ }^{3,4}$

The current study does not tell us exactly how much earlier recurrences are identified in patients with more intensive follow-up compared with less intensive follow-up given all patients were followed by relatively intensive 
follow-up regimens. Median time to recurrence in the present series was between 12 and 18 months. In a large United States series, median time to recurrence following potentially curative resection of gastric cancer was about 1 year. $^{5}$

When recurrent gastric cancer is diagnosed, we have effective therapies although the magnitude of survival benefit is modest. The median survival of patients with advanced or metastatic gastric cancer is less than 6 months with best supportive care. ${ }^{6}$ In a meta-analysis of 3 randomized trials of combination chemotherapy versus best supportive care, combination chemotherapy improved mean survival by about 6 months. ${ }^{7}$ The REAL- 2 trial compared 4 combination chemotherapy regimens (ECF, ECX, EOX, and EOF) in more than 1000 randomized patients with advanced esophagogastric cancer (about $40 \%$ were gastric cancers) and determined these regimens to be equally efficacious with median survivals ranging from 9.3 to 11.2 months. ${ }^{8}$ For HER2-positive advanced gastric cancers, the addition of trastuzumab (a monoclonal antibody targeting the HER2 receptor) to chemotherapy increases median overall survival by 2.7 months.

While we have randomized trials demonstrating that combination chemotherapy increases overall survival in patients with advanced gastric cancer, it is unknown if more expeditious institution of chemotherapy following intense surveillance will improve outcomes compared with institution of chemotherapy when recurrent disease has progressed to causing symptoms. ${ }^{9}$ For resectable gastric cancer, perioperative chemotherapy can presumably eradicate microscopic residual disease in a minority $(10 \%-$ $15 \%$ ) of patients, but combination chemotherapy rarely cures macroscopic disease. ${ }^{10}$ Thus, one may extrapolate that chemotherapy may be more effective when used against decreased volume of disease, but this conjecture requires further evidence. ${ }^{11}$

Given the lack of data on the effectiveness of more intensive follow-up of gastric cancer patients following resection, there are significant differences in the recommendations of various groups. The European Society for Medical Oncology (ESMO) clinical recommendations for the follow-up of gastric cancer state "there is no evidence that regular intensive follow-up improves patient outcomes, [and] symptom-driven visits are recommended for most cases." 12 However, many patients are uncomfortable with minimal or no follow-up. The National Comprehensive Cancer Network practice guidelines for gastric cancer recommend a history and physical examination every 3-6 months for 1-3 years, every 6 months for 3-5 years, and then annually. Complete blood count (CBC), chemistry profile, radiologic imaging, and endoscopy are recommended as clinical indicated. ${ }^{13}$ Ultimately, the decision regarding the intensiveness of follow-up is left to the treating physician after discussion with the patient.

Hopefully in the future, biomarkers for gastric cancer will be developed that identify both primary disease and recurrences at a very early stage. That future may not be too far off. Haber and colleagues at Massachusetts General Hospital have developed a highly sensitive assay for the identification of tumor cells (CTC-chip) in peripheral blood that detected tumor cells in nearly all patients with metastatic solid tumors as well as in $42 \%$ of patients with earlystage prostate cancer. ${ }^{14,15}$ Diaz and colleagues at Johns Hopkins Medical Institutions have developed a method of for quantifying circulating tumor DNA in peripheral blood using real-time quantitative PCR and BEAMing technology. ${ }^{16}$ Of 16 patients found to have persistent circulating tumor DNA following potentially curative surgical resection, 15 of 16 patients ultimately recurred. Early detection of primary disease when it is localized and highly curable will make the detection of recurrent disease a topic of much less frequent debate.

\section{REFERENCES}

1. Tjandra JJ, Chan MK. Follow-up after curative resection of colorectal cancer: a meta-analysis. Dis Colon Rectum. 2007;50: 1783-99.

2. Eom BW, Ryu KW, Lee JH, Choi IJ, Kook MC, Cho SJ, et al. Oncologic effectiveness of regular follow-up to detect recurrence after curative resection of gastric cancer. Ann Surg Oncol. 2010. doi:10.1245/s10434-010-1395-3.

3. Smith DL, Elting LS, Learn PA, Raut CP, Mansfield PF. Factors influencing the volume-outcome relationship in gastrectomies: a population-based study. Ann Surg Oncol. 2007;14:1846-52.

4. Jemal A, Siegel R, Xu J, Ward E. Cancer statistics, 2010. $C A$ Cancer J Clin. 2010;60:277-300.

5. Bennett JJ, Gonen M, D'Angelica M, Jaques DP, Brennan MF, Coit DG. Is detection of asymptomatic recurrence after curative resection associated with improved survival in patients with gastric cancer? J Am Coll Surg. 2005;201:503-10.

6. Pisters PWT, Kelsen DP, Teper JE. Cancer of the Stomach. In: DeVita VT, Lawrence TS, Rosenberg SA, editors. Cancer: Principles \& Practice of Oncology. 9th ed. Philadelphia: Lippincott Williams \& Wilkins; 2008;1741-94.

7. Wagner AD, Grothe W, Haerting J, Kleber G, Grothey A, Fleig WE. Chemotherapy in advanced gastric cancer: a systematic review and meta-analysis based on aggregate data. J Clin Oncol. 2006;24:2903-9.

8. Cunningham D, Starling N, Rao S, Iveson T, Nicolson M, Coxon $\mathrm{F}$, et al. Capecitabine and oxaliplatin for advanced esophagogastric cancer. N Engl J Med. 2008;358:36-46.

9. Whiting J, Sano T, Saka M, Fukagawa T, Katai H, Sasako M. Followup of gastric cancer: a review. Gastric Cancer. 2006;9:74-81.

10. Cunningham D, Allum WH, Stenning SP, Thompson JN, van d, $\mathrm{V}$, Nicolson M, et al. Perioperative chemotherapy versus surgery alone for resectable gastroesophageal cancer. $N$ Engl $J$ Med. 2006;355:11-20.

11. Bang YJ, Van CE, Feyereislova A, Chung HC, Shen L, Sawaki A, et al. Trastuzumab in combination with chemotherapy versus 
chemotherapy alone for treatment of HER2-positive advanced gastric or gastro-oesophageal junction cancer (ToGA): a phase 3, open-label, randomised controlled trial. Lancet. 2010;376: 687-97.

12. Jackson C, Cunningham D, Oliveira J. Gastric cancer: ESMO clinical recommendations for diagnosis, treatment and follow-up. Ann Oncol. 2009;20 Suppl 4:34-6.

13. NCCN Clinical Practice Guidelines in Oncology v.2.2020: Gastric Cancer. 1-1-2010. http://www.nccn.org/professionals/physician_ gls/f_guidelines.asp.
14. Nagrath S, Sequist LV, Maheswaran S, Bell DW, Irimia D, Ulkus $\mathrm{L}$, et al. Isolation of rare circulating tumour cells in cancer patients by microchip technology. Nature. 2007;450:1235-9.

15. Stott SL, Lee RJ, Nagrath S, Yu M, Miyamoto DT, Ulkus L, et al. Isolation and characterization of circulating tumor cells from patients with localized and metastatic prostate cancer. Sci Transl Med. 2010;2:25ra23.

16. Diehl F, Schmidt K, Choti MA, Romans K, Goodman S, Li M, et al. Circulating mutant DNA to assess tumor dynamics. Nat Med. 2008;14:985-90. 